duces sufficient shock on the circulatory and nerve apparatus to quickly atrophy growing uterine myo. mata. The effect of ligating the uterine arteries as they course through the broad ligament is to atrophy the endometrium rapidly-checking endometrial secretions and hemorrhage. The pelvic floor is preserved intact by this operation, obviating the danger of vaginal hernia.

In a vaginal hysterectomy made by Dr. Lucy Waite with my assistance, a vaginal hernia followed several months after the operation. This operation brings on the menopause rapidly. It followed in two months in one case. The operation leaves a functionless, atrophic, normal positioned uterus, which so far among our patients has given no trouble. This report is based on thirty operations made during the last twenty-six months. In several of the cases I assisted Dr. Lucy Waite and Dr. Bertha Van Hoosen, and include them in the report. The utility of the operation consists in the following points :

1. It checks hemorrhage.

2. It arrests menstruation.

3. It elevates the uterus.

4. It shrinks the uterus.

5. It avoids the removal of uterine myoma.

6. It avoids vaginal hernia.

We have proved that the operation is suitable to every one of the above six propositions. Dr. Van Hoosen reports that not one of the cases in which I assisted her in operating has menstruated. I know of not a single case in which Dr. Waite and I operated where menstruation continued. We removed the appendages and tied the uterine arteries on a patient 37 years old, with a myoma extending above the pelvic hrim. Three months later the tumor was decreased one-half; eight months later the uterus is what I should term almost normal in size. The operation shrinks the metritic uterus, which nearly always accompanies suppurating appendages. It shrinks the endometrium so that excessive secretions are checked. I have proved the shrinking or atrophying of the large, hard, metritic uterus, time after time, by subsequent examination. The operation elevates the retroverted uterus and maintains it in position. It is superior to hysteroexey, as it leaves a movable uterus.

Two months ago I operated with Dr. Verity, of Chicago, on a case with diseased appendages, and with a large, hard, retroverted metritic uterus. We removed the appendages and tied the uterine arteries almost down to the cervix. Eight weeks subsequent to the operation I examined the patient and the uterus was in distinctly normal position; it was movable and had shrunk at least one-third in size. Two objections might be raised against the operation:

1. The danger of gangrene.

2 . The ligation of so much tissue.

I do not consider it an objection that one might penetrate the bladder with a ligature armed needle, or a ureter might be included in the ligature, as any other operation is encumbered with similar dangers. The idea that the ligatures might become infected and produce subsequent fistule, is not a serious objection. I would suggest that the ligature which includes the broad ligament should not include the Fallopian tube with its mucous membrane, as that might enhance the chances for suppuration. The needle could be passed just beneath the tube. This method ought not to protract the operation more than ten minutes. In the thirty cases, one patient died from the results of the operation. The patient was a weak, worn-out woman of 37 , who had a myomatous tumor for some six years. The broad ligament was tied in sections and the tumor shrank so rapidly in four days that it produced a kink in the bowel by dragging on it. The bowel was not completely obstructed. The patient went on well for four days, after which she suddenly sank in a few hours, without many symptoms. An autopsy revealed the fact that in four days the tumor had shrunk enormously, and a kink was found in the bowel. The debility of the patient and the worn-out nervous centers were to blame for this death. The other death among the thirty cases was due to urinary suppression on the sixth day in a woman who had almost flowed to death during six years with a large uterine myoma. The case progressed well for six days after the operation, when the urine ceased to be secreted and she died about thirty-six hours later.

\section{THE SURGICAL TREATMENT OF INJURIES OF THE HEAD.}

TREPHINING FOR CLOT AND PRESSURE SYMPTOMS-NO FRACTURE.

BY R. E. HAUGHTON, M.D.

Formerly Professor of Surgical Pathology, Operative and Clinical Sur gery, in Central College of Physicians and Surgeons Indianapolis Member of Ameriean Medical Association, of Mississippi Valle Medical Association, of Texas State Medical Association, exresident Indiana State Medical Association and Hor MIDLAND, TEXAS.

In view of the great apparent progress in cranial surgery, in many forms of disease as microcephalus, idiocy, hydrocephalus, tumors, compression, clots of blood, cases where a transmutation of structure may take place, viz., hyperplasia of connective tissue, neuroglia of Virchow or glioma, I report a case to show what may be accomplished in the earlier stages of gevere injury in aid of a final recovery. We must in some degree comprehend the structure and anatomy of the relations of the brain, how protected by its membranes, its bony case, its supply of blood within and without, the sources of blood supply internal and external; and comprehend the dilatation and contraction of the vessels under vasomotor influence, the reflex irritation upon vessels, the difference of cause in coma of compression and cause of irritation which may be reflex or direct, the relation of blood supply to connective tissue, before we are in any sense, in a condition to comprehend the many varieties of symptoms which present themselves, as in disease or injury, which progresses to very serious results. The result of experimentation upon the brains of mammalia with organizations much inferior to man, leave many questions as yet unanswered in regard to the human brain.

The diseases of motility, speech, sensibility and special sense all require a large comprehension of a very large number of pathologic facts which have been so far utilized in connection with the more recent developments in histology, so that to some extent a clinical summary is being reached which enables us in a measure to make out a clinical or surgical diagnosis with some degree of certainty.

Again, cerebral lesions are revealed by symptoms which when classified, enable us with some certainty to reach a diagnosis, for instance, we find a large 
group of symptoms which are cerebral or cephalic, which are an entire departure from normal conditions and which, though beginning as to brain, produce disturbance of function of one-half of the body. Consciousness is in entire abeyance, and we have excitement, incoherence, delirium, or we have the oppo site conditions of entire absence of brain power in any manifestations of intellect, or if any brain intelligence is manifest there is a complete abolition of motor power by which the senses operate externally. Paralysis of motion and sensation, especially on one side of the body, indicate physiologically, the crossing of fibers as they pass to the cord by way of the medulla oblongata, so that we begin to locate and classify symptoms. Irritation of motor functions, shown in stiffness, spasms or rigidity, clonic or choreic convulsions, with derangement of coördination or tremors of a limb of one-half of the body. The peculiar group of cerebral paralyses are less of ten produced by hyperemia or anemia of certain brain regions than by localized lesions of a sudden or acute character, viz., hemorrhage, serous effusion, inflammation and results, or hemorrhagic clot and compression because of a more or less widely diffused injury. In addition to these in immediate origin are the results of primary and secondary lesions causing arrest of motor or sensory impulses, because the paths or lines of transmission are injured, compressed or destroyed. The doctrine of localization of function has done much to clear up our ideas as to the relations of certain, local and definite functions residing in a special part of the brain, and thus following out the demonstrated relations of function we are enabled to clear up the diagnosis.

It is well recognized that the hemiplegic type of paralysis upon the opposite side to cerebral lesion is due to disease of motor centers as corpus striatum and lenticular nucleus, or to localized lesions of the cerebral lobes involving the peduncles and ponsvarolii. The paralysis of one-half of the body appears in the muscles of the limbs, the extensors and the contraction of the antagonists. The muscles of the paralyzed side generally undergo no noteworthy change in electric sensibility.

This and much more might be said in reference to the varying conditions which may obtain in case of an injury which may be first, concussion, followed at once by symptoms of compression, which may and often does require surgical diagnosis and operative measures to relieve the compression, as in the case I am about to present:

Case 1.-Mr. W., a citizen of Midland and a cattle man, had gone out to his ranch to look after his cattle. He rode a horse which was not well broken, and for some reason, not known the animal reared and threw his rider, his head coming in contact with a pile of stone, which struck him about the superior margin of the left parietal bone near the parietal protuberance, producing a contusion of the scalp and also cut down to the bone. His skull was supposed to be fractured, by the physician who was called out to see him and he adhered to this opinion. On the second day Mr. W. was brought to Odessa and from there by train to Midland, a distance of about forty miles. During the time which elapsed from his injury, he was unconscious, paralyzed on the entire right side, stertorous respiration, pulse slow and labored, with difficult respiration at times and spasmodic action of the muscles of the same side as the injury, viz. left. Pupils dilated at first were afterward contracted. Functions of bladder and bowels arrested, except a stillicidium or dribbling from the bladder.

Diagnosis or Summary.-Concussion with compression of brain as shown by all the leading symptoms. Shock had been marked from the first, yet a sufficient reaction was secured to move him as before mentioned, as no operative procedure could be instituted on the ranch. On Feb. 19,1893, I was called into consultation with Drs. E. and C., just after night as he reached Midland, at nearly dark. The decision was reached that in an operation to determine whether there was fracture a depressed fracture, it was necessary to elevate the bone and relieve the pressure as it was a case of compression though no fracture could be found. It was also determined by the consultation to operate for two reasons: 1, to determine as to the existence of a depressed fracture; 2 , if this did not exist to trephine for the purpose of removal of a coagulum of blood, as it was assumed this also might exist from the nature of the injury, and as well from all the symptoms.

On the following morning February 20, we saw the case and proceeded to make an exploratory operation as to fracture, dissected up the scalp for the purpose and clearly demonstrated that no fracture existed. Then we at once proceeded to trephine at the point of contusion to find the cause of compression. We removed a small circle of bone, including both tables and upon its elevation the blood began to well up into the opening, very much as water wells up in to a well when you reach it. The blood had not coagulated as it was occasioned by the rupture of divisions of the middle cerebral artery or parietal ascending branch. The duramater was carefully protected while using the trephine hence the blood was from the artery, the middle "ascending parietal artery." There was a large amount of blood which was taken up by small sponges as it filled the opening. When this had been removed there seemed no clot in sight or on touch. The dura mater was intact so that we proceeded to close and dress the wound antiseptically. One suture was placed by a silver wire at the apex of the flaps and the wound had an antiseptic solution of peroxid of hydrogen and bichlorid of mercury in water, 1 to 2000 with light compress and bandage. 'The wound healed readily without suppuration, and the symptoms of compression slowly passed away. The patient after careful nursing, has recovered so he is working and transacting business, and was able to ride and walk in a few weeks after the operation. Since his recovery he went to Buffalo, New York, to the Sur gical Institute to consult (as he believed) abler counsel than he could find on the plains. He came as he went, being informed that so far as surgery was concerned all had been done that could be done. I had an opportunity to determine his condition as he rode out in a buggy as soon as able, and one man who frequently took him out said to me: "He knows a fat steer and its value, and whether he owns it as quickly as he ever did, as I tested bis capacity in that direction."

Some questions now arise as to the diagnosis, and in explanation of some if not all the principal facts in this case, it should be remarked here that we have had this year three cases of injury in the head, two of them recovering without any operative procedure, being only concussion of the brain. All of these cases were produced by falls from horses, two occurring in the night and the one here recorded in the day time.

The physiologic and pathologic fact existed of hemiplegia from the injury. This fact indicates, that compression of the fibers or cells of the left parietal convolution as they pass down in to the medulla are transferred to the opposite side and (vice versa) when the injury is upon the other side. This was the principal fact which guided in this operation and which coincided with the second fact that the ("pt-de-appui") or site of injury was on the left, hence other things being equal we should find the compression under the injury.

Again, when we come to make up a diagnosis as to the clonic convulsive action of the same side of the injury, "the hemiplegic type of paralysis upon the side opposite to the cerebral lesion is due to disturbance of the motor ganglia, corpus striatum and lenticular nucleus, or it may be due to localized lesions of the cerebral lobes involving the peduncles and pons varolii."

Again, when hemiplegia occurs upon the same side as the cerebral lesion, a center is found in the motor 
:ganglia of the opposite side with alteration in the lobes of this side, and it may be with edema or pressure from blood in the parenchyma of the structure of the opposite side. We had a partial hemiplegia on the same side of the injury, but it was not persistent or permanent. This was accounted for from the fact that the concussion by a contra coup may hav $s$ produced small foci of blood deposit and induced the condition of the side injured, or incomplete hemiplegia with startings or twitchings and spasmodic action of the muscles of the arm and leg of the injured side.

I have said the patient was trephined at Midland, and has slowly recovered, regaining such measure of power, intellectually and physically, as to walk about town with a cane yet with irregular, somewhat spasmodic action of the muscles of the limbs below, showing that he has not yet fully recovered the motor control of certain sets of muscles. I believe he will continue to improve in this direction until a fair use of the muscles may be regained.

Later, Jan. 1, 1895, he is still improving, and now superintending and building a large four-story hotel.

Surgical appreciation of methods and operations in traumatic and intra-cranial lesions without fracture. We have already come to a conclusion as to operative methods in cases of fracture, especially with depression of tables and clots of blood which may form in such cases, as in elevation of depressed bone and removal of loose or detached fragments. That is, relieve (brain pressure.) This leads us to another step. What shall be done with cases of intra-cranial lesions (traumatic) in which no fracture exists, as was clearly demonstrated in this case? Evidences of compression were clear, yet no fracture existed. What could cause compression? Two conditions clearly; effused blood which may be found 'beneath the injury of' the surface, and points of effusion, (ecchymosis punctatæ diffusa) and, finally, fracture by contra coup might arise where lesion or force is sufficient. While cerebral localization may not and most probably will not always indicate the spot at which the cranium may be trephined, nevertheless in such a case as I have here described the demand is none the less as imperative as in cases of depressed fracture, as compression by extravasation of blood exists in both cases and in the first class of cases, bone is also depressed. What are the facts in cases where post-mortem examinations have been made?

1. Death occurs in all ages and conditions from such head injuries, without fracture of the skull, and the facts thus shown prove that a large per cent. of cases dying from brain fever and meningitis, especially in children, are the result of inflammatory action about a clot, producing abscess or softening due to some degeneration of injured cerebral tissue. Hence we may deduce a rule of action, that because these cases are not found accompanied by fracture there is certainly no good reason why they should not receive :surgical attention, as they are amenable to treatment, as this case shows. Reports of cases, as I have gathered them up, show that the most grave cerebral lesions, manifested by very grave and serious symptoms, are produced without the slightest solution of continuity in the cranial structure and sometimes with no laceration or contusion of the scalp. Concussion of the brain, as a whole, may eventuate in a slow process of inflammation, softening or abscess and death some time after the injury and when to all appearance the patient had seemingly recovered. I have a case now under observation who received a severe concussion, remaining unconscious for several days, but finally slowly recovering save a little unsteadiness of gait and excitability of manner. He was at first paralyzed in some of the muscles of the right side. No mark or coutusion on the head or any part of the body.

The symptoms of pressure are induced by the effusion of the blood between the dura mater and skull as in this case, and which was fluid and not a clot, and the pressure symptoms were of a most severe and profound nature, hemiplegia of one side and par. tial of the other side, stertor, coma, depression and partial arrest of most of the vital functions, inability to swallow and many others, making up an entire aggregate of symptoms which threatened the life of the person.

Again, a rupture of a larger or smaller trunk or branch of the middle, meningeal or cerebral arteries would produce sudden and great compression and possible death, and yet there has been no fracture or injury external, but as in this case profound disturbance of all the nervous and vascular areas. What shall be done with such cases? Formerly they were bled, purged, blistered and tortured by an experimental medication till the patient was dead, or, with a vital force which would not succumb to the injury, aided by the bombardment of remedies got well in spite of disease and the doctors. The evidence of compression of cortical centers may be determined with some degree of certainty by a comparison of symptoms contrasted as here stated. I quote from the "Encylopedic Index of Surgery :"

concussion.
1. Insensibility more or less com-
plete.
2. Respiration feeble.
3. Pulse weak, irreguiar and fre-
quent.
4. Special senses dull.
5. Pupils variable aud sensitive to
light.
6. Nausea as recoyery goes on.
7. Bowels relaxed. sphincters not
paralyzed.
8. Bladder can expel the contents.
9. Comes on instantly, but passes
off slowly.

COM PREssion.
1. Total insensibility.
2. Respiration stertorous, slow,
pufyy.
3. Pulse slow, full, labored.
5. Pupils widely dilated or one
dilated and the other con-
tracted.
6. Stomach insensitive.
7. Sphincters paralyzed and bow-
els torpid.
8. Bladder paralyzed.
9. Does not appear at first but
grows worse. The two conditions usually coëxist, but the compression is found to exist and hence concussion may pass into compression as the latter term means pressure, and paralysis is usually taken as a distinguishing mark-a diagnostic sign of compression. The cause of compression is here in such cases with no fracture a hemorrhage, and we should know where it is most likely to occur and the fact of compression make us cast about to determine the location of hemorrhage. If it is an injury done to the medulla, cerebral artery or its branches we can determine readily enough from the nature of the injury, as blood may be deposited between the cranium and dura mater, or if the latter is broken or injured, or if not so, sooner or later we have compression of the cortex and also we have pressure upon the ventricles and cerebrospinal fluid and thus a general disturbance by pressure which must be relieved if possible. These conditions imply extravasation of blood within the cranium, contusion or laceration and these conditions are found upon the surface of the dura mater. In the cavity of the arachnoid, on the brain surface in the substance of the brain as by foci, or in the ventricles, especially the lateral and at the lenticular 
nucleus. It may occur in the corpus striatum, thal. amus opticus, and if in the cortical structure near the surface may, as the blood continues, force its way through the cerebral matter, uplift the pia mater or find its way into the cavity of the arachnoid. The most common location of hemorrhage is the corpus striatum and thalamus opticus in apoplexy, or from disease of vessels, and rarely in the white structure, viz. corpus callosum. Large amounts of blood will occasion tumefaction and softening of the cerebral mass and flattening of the convolutions of the hemisphere which is the seat of lesion; before an examination may be made it is suspected. Hence a rule of action now is to trephine promptly so that these graver lesions shall not have time to occur, as well as to remove the primary cause of trouble, the blood, whether it be a clot or fluid as pressure exists, and the patient has no power of continuance unless relief can be offered by a removal.

I shall not attempt to follow the blood and tissue changes as it would not be competent, but only to indicate the procedure and the promptness with which it should be done. The surgeon who fails to offer his patient the relief which such a procedure as trephining and removal of so much of cranial bone as may give relief to the pressure, and removal of the clot and fluid débris to the great relief of the existing pressure upon the brain, either has not comprehended what it is to take in the situation by a summary, or is too temporizing to be a safe surgeon. Suffice it to say that this case was operated upon Feb. 21, 1893, and is now and has been for a year or more walking around our streets in the transaction of his business, and while he does not walk as steadily as I do, yet he is far better than a dead man and gives promise by improvement that he may yet recover fully, due to the prompt use of the trephine or chisel in removal of blood pressure.

The Technique of the Operation.-The method to be pursued in microcephalus and in any condition of the brain requiring an operation is that it shall be done as quickly as possible to give sure and prompt relief to the compressed organ. In cases of injury without fracture but with sufficient evidence to require an operation to be made, the surgeon should resort to one of several methods in exposing the bone and dealing with the scalp. It may be a long $U$ or rather a horse-shoe flap which has been preceded by a running stitch around the region of the flap beyond it, so as to control the bleeding from the scalp. The horse-shoe flap perhaps is preferable, yet if by any preference of education or otherwise, he may resort to the crucial incision, and expose the bone. The trephine may be used in one or more places, and then by cutting forceps cut away the interfering structure so as to gain room and relieve pressure. This should be done, cutting as much or as little as the special case in hand may demand.

It may be found in compression by blood or clot that the blood is found beneath the dura mater or external to it. Then the incision of the dura mater may require to be done to reach the blood, or if there is evidence that there is blood or pus beneath the surface of the dura or pia mater or within the arachnoid spaces, the pressure should be relieved. It may be difficult to determine some cases, yet ordinarily the diagnosis can be made and the operation should follow. In fact, I hold that it is imperatively demanded of the surgeon in the face of unconsciousness or coma, with paralysis of one or both sides of the body, a slow and labored pulse and more or less difficulty of respiration, relaxation of the sphincters of the body, etc., to operate, and expect beside to benefit and probably save his patient. The technique should be great cleanliness and antiseptic precautions with as much protection to all the delicate structures as may be possible. Antiseptic gauze, iodoform, boracic acid solution ( 1 to 25 to 50 ) bichlorid mercury solution ( 1 to 3000 to 5000) or more, light bandage and compress after parts are externally adjusted. The patient may not be able to take anything until consciousness returns, then light unstimulating diet, quiet and rest in a room well ventilated at temperature of 70 degrees.

Conditions.-As a summary of propositions in such cases:

1. Cases of coma by compression, as distinguished from coma by irritation, which may be reflex or direct. We must decide a case, as one of compression, by some intercranial cause before an operation is surgically admissible.

2. That coma from reflex conditions and affecting vsssels, and producing coma by irritation, or coma by contraction of vessels, and as a result vascular anemia, must be distinguished from the former.

3. There shall be no fracture in the cases harein considered.

4. We may have concussion and final compression of brain from rupture of vessels, accumulation of blood and pressure symptoms.

5. Given such conditions, what surgical procedure is required if any (except in second proposition), but found in first, second, third and fourth propositions. Answer to these propositions: Operation-by necessity-except in proposition 2 .

\section{THE SURGICAL TREATMENT OF INJURIES OF THE HEAD.}

BY CHARLES B. PARKER, M.D., M.R.C.S., ENG. CLEVELAND. OHIO.

The treatment of head injuries is a subject of general interest. Such injuries occur in the practice of every medical man. With the exception of such general remedies as rest, ice, cathartics and sedatives their treatment is necessarily surgical, and when successfully carried out furnish a good test of the mastery of the technique of aseptic and antiseptic methods.

No injury of the head can be considered trivial, for the simplest one may lead to fatal consequences. The cause of this results anatomically from the wellknown close structural connection between the coverings and contents of the brain, and pathologically, in the fact of direct or embolic germ infection. Anatomically we have a rather thin skin connected to the aponeurosis of the occipito-frontalis muscle by a very dense cellular structure, the fibers of which have something of a ligamentous strength and inclose a peculiarly vascular adipose tissue with which the vessel's walls are intimately connected. The bone is not solid-an outer tough and an inner brittle table, and between, the vascular diploë; beneath these the dura mater. You know how intimately the dura is attached to the inner surface of the calvarium if you have ever attempted to remove it post-mortem. The dura mater is more dense than pericranium or periosteum; it is a fibro-serous membrane. You know 